\title{
Incident-to Billing for Pharmacists
}

\author{
Eric Dietrich, PharmD, BCPS, CPC-A, and John G. Gums, PharmD, FCCP
}

\begin{abstract}
SUMMARY
The inclusion of pharmacists on care teams has been shown to improve clinical and economic health outcomes. However, a significant barrier to the widespread incorporation of pharmacists into care teams is the ability to financially support the salary of the pharmacist. A mechanism to improve the ability of pharmacists to generate clinical revenue already exists in the form of incident-to billing, although there remains considerable uncertainty regarding the criteria for incident-to billing and specifically how pharmacists can use this model to capture revenue for clinical services. In this article, we discuss incident-to billing criteria as it pertains to outpatient clinics, common misconceptions related to incident-to billing, and how clinical pharmacists may use this mechanism to generate revenue for the clinical services they provide.
\end{abstract}

J Manag Care Spec Pharm. 2018;24(12):1273-76

Copyright $\odot 2018$, Academy of Managed Care Pharmacy. All rights reserved.

$\mathrm{P}$ harmacists are playing increasingly larger roles in the outpatient setting by working with physicians and patients to provide medication management services for numerous chronic disease states. The benefits of this collaborative relationship, often called physician-pharmacist collaborative management (PPCM), are well documented for chronic disease states, namely hypertension and diabetes, with improvements in attainment of goal blood pressure, hemoglobin Alc, and cholesterol markers. ${ }^{1}$ In addition to improvements in clinical outcomes, PPCM has been shown to decrease health care costs. Three separate studies have shown that including pharmacists in the care of patients with hypertension, hyperlipidemia, or diabetes resulted in an average net savings of \$918-\$3,556 per beneficiary per year to the health plan..$^{2-6}$ Furthermore, a report to the U.S. Surgeon General from the Office of the Chief Pharmacist of the U.S. Public Health Service noted that for every $\$ 1$ spent on pharmacy services an average of $\$ 10.07$ was saved in health care spending. ${ }^{7}$ Recognizing these benefits, the Centers for Medicare \& Medicaid Services has recently supported an expanded role for pharmacists in direct patient care, noting specifically that pharmacists can help improve patient access to care. ${ }^{8}$

Despite the benefits of PPCM, widespread integration of pharmacists into care teams remains limited, most notably due to financial barriers. Because of the lack of national provider status, pharmacists have not been able to directly bill for their services at a level commensurate with their knowledge and skills. This financial barrier effectively limits the ability to financially sustain a collaborative model with other health care providers to improve patient outcomes and patient access to high quality health care. As such, many outpatient centers have been hesitant to take on the financial burden of hiring a clinical pharmacist despite the documented benefits of PPCM.

Historically, in-office visits and services rendered by a clinical pharmacist were billed exclusively at Level 1 (or Current Procedural Terminology [CPT] Evaluation and Management [E/M] code 99211, which typically is billed when 5 minutes of very simple clinical services are provided) regardless of the amount of time spent by the pharmacist, the complexity of the visit, or the number of interventions made. Since the average Medicare reimbursement for this level of service is approximately $\$ 20$, this is not a financially sustainable long-term model that would provide sufficient salary offset for most clinical pharmacists, especially when including overhead costs and billing expenses that, in many cases, may exceed the actual reimbursement for the visit. However, if a pharmacist was able to receive reimbursement for services at a higher level (such as with CPT E/M codes 99213 [Level 3] or 99214 [Level 4]), which would be more consistent with the level of services provided and similar to the level of service that would be billed by a physician, then the long-term sustainability and feasibility of the service becomes significantly more attainable (Table 1), even when real-world considerations such as overhead costs are included.

One such mechanism to improve the ability of pharmacists to generate clinical revenue already exists in the form of incident-to billing. However, there remains considerable uncertainty regarding the criteria for incident-to billing as it pertains to pharmacists and how pharmacists can use this model to capture revenue for clinical services. In this article, we discuss incident-to billing criteria as it pertains to outpatient clinics and clinical pharmacists, common misconceptions related to incident-to billing, and how clinical pharmacists may use this model to generate revenue for the clinical services they provide.

\section{Defining Incident-to Billing}

Medicare defines incident-to billing as "services that are furnished incident to physician professional services in the physician's office." Further, these "services are billed as Part B services to your carrier as if you [the physician] personally provided them, and are paid under the physician fee schedule." Medicare provides these further requirements:

- The services must be part of the patient's normal course of treatment, during which a physician personally performed the initial service and remains actively involved in the course of treatment. 
TABLE 1 Number of Visits Required to Break Even ${ }^{a}$

Level of Service, $\$$

\begin{tabular}{|c|c|c|c|}
\hline \multirow[b]{2}{*}{ Visit Frequency } & \\
\hline & Level 1 & Level 3 & Level 4 \\
\hline Average Medicare reimbursement & 21.96 & 74.16 & 109.44 \\
\hline Visits per yearb & $6,374.00$ & $1,997.00$ & $1,355.00$ \\
\hline Visits per week & 122.60 & 38.40 & 26.10 \\
\hline Visits per day & 24.50 & 7.70 & 5.22 \\
\hline
\end{tabular}

Source: Medicare Physician Fee Schedule Look-up tool (https://www.cms.gov/apps/ physician-fee-schedule/license-agreement.aspx).

${ }^{a}$ Assumes $\$ 0$ overhead costs and a salary plus fringe of $\$ 125,000$.

${ }^{b}$ Assumes 52 weeks per year.

- The supervising physician must provide direct supervision. For group providers, any physician member of the group may be present in the office to supervise.

- The patient record should document the essential requirements for an incident-to service. ${ }^{9}$

While most commercial payers follow the same billing rules established by Medicare, they are not required to do so, and one would have to confirm with each individual carrier their specific incident-to billing rules.

There are important points to consider within this definition. First, it is specifically noted that the physician bills Medicare Part B as if they were the one performing the service. It is not the pharmacist who is billing Medicare; instead, the pharmacist is furnishing the service incident-to the professional service of the physician. However, since pharmacists are performing the clinical service, they are eligible to receive partial to full financial credit when the practice submits for reimbursement from Medicare by including the pharmacist as a secondary provider on the encounter sheet or superbill. Inclusion as a secondary provider within the clinic's billing system will allow tracking of revenue to the actual service provider, while the physician remains the billing provider.

Because the supervising physician remains the billing provider, he or she will receive the associated work relative value unit (wRVU) credit for the visit, which is incentive for some physicians to collaborate with pharmacists under this type of model, since in most settings, physician bonuses are tied to wRVU targets and collaboration would increase the wRVUs generated by the physician. Physician liability may also be reduced by working collaboratively with a medication expert to ensure that therapeutic treatment regimens are used safely and are monitored appropriately. Contractual agreements, such as a Memorandum of Understanding, may be required between the pharmacist and physician or physician group in order to clearly outline how the pharmacist will receive financial credit when providing clinical services, since the pharmacist will not be the billing provider.
Second, the physician must have performed the initial patient service before subsequent visits by the pharmacist to be eligible for incident-to billing, which provides the physician an opportunity to establish the diagnosis and develop a treatment plan for the patient (another requirement for incident-to billing). Implementation of a signed collaborative practice agreement (CPA) between the physician and the pharmacist provides specific criteria for future services provided by the pharmacist and is an established and accepted mechanism to fulfill the intent of the Medicare incident-to billing provision. More discussion of CPAs will be presented later, but it is important to emphasize that CPAs require physicians to direct patient care, including determining which patients are seen by the pharmacist, which conditions or medications the pharmacist will manage, and what degree of responsibility the pharmacist will have.

Finally, the physician must provide direct supervision of the pharmacist performing the incident-to service. There are 3 different levels of supervision ${ }^{10}$ : general supervision requires the physician to provide overall supervision of the service, but the physician is not mandated to be at the place of service; direct supervision requires the physician to be at the place of service and be immediately available to assist but is not required to be in the room where the service is provided; and immediate supervision requires the physician to be physically present in the room where the service is being provided. The requirement of direct supervision for incident-to services allows the pharmacist to work independently of a physician, allowing that physician to continue conducting clinic visits thereby helping to improve patient access to care. Direct supervision also allows the pharmacist and physician to practice independently and collaboratively at the highest level of their training and education, but when circumstances arise that are outside the training and experience of a pharmacist, the physician is immediately able to assist.

\section{Qualifying for Incident-to Billing}

Administrators may suggest that pharmacists are not eligible to perform incident-to billing because they are not classified as a "provider" by Medicare Part B and are not specifically mentioned in Medicare rules as being able to perform incident-to services. ${ }^{10}$ To clarify the potential role of pharmacists in the incident-to billing model, the American Academy of Family Physicians (AAFP) directly queried Medicare, asking if pharmacists were eligible to provide clinical services in an incident-to manner. ${ }^{11}$ Notably, AAFP reviewed the Medicare Benefit Policy Manual, specifically chapter 15 sections 60 (A) and 60.1 (B), and could not find anything that would exclude pharmacists from meeting requirements for incident-to billing as auxiliary personnel. Medicare responded to AAFP, agreeing that pharmacists are eligible to provide clinical services in an incident-to billing model provided that all additional 
requirements of incident-to billing were met. ${ }^{11}$ In addition, Medicare stated that the services provided must meet all other applicable state laws. Therefore, although Medicare billing rules do not specifically classify pharmacists as providers or as a specialty able to perform services incident-to, Medicare has officially stated that pharmacists meet all required eligibility criteria in order to perform services under an incident-to model provided the pharmacy services are within state scope of practice laws.

\section{Scope of Practice Laws}

Each state individually defines the state scope of practice for pharmacists, which has led to a wide range of practices throughout the United States, making it more difficult to uniformly apply the AAFP response from Medicare regarding the ability of pharmacists to provide services in an incident-to model. As of 2016, 48 states had legislation explicitly authorizing CPAs or collaborative drug therapy management. ${ }^{12}$ Notably, Tennessee, with recently approved state legislation, has become the second state (behind Washington in 2016) to formally recognize pharmacists as providers in state-run health plans.

Despite the lack of a consistent definition at the state level regarding scope of practice, the more uniform presence of legislation across states allowing pharmacists to enter into CPAs may provide a mechanism to satisfy the Medicare requirement that services provided by pharmacists meet applicable state laws. The Centers for Disease Control and Prevention have published a document outlining potential steps for creating and implementing CPAs to assist pharmacists in this arena. ${ }^{12}$

\section{Use of CPAs}

Further, the use of CPAs meets additional requirements of incident-to billing. According to Medicare requirements, the physician must first meet with a patient and develop a treatment plan. At this point, a CPA could be executed, outlining the role and responsibilities of the pharmacist in providing further care for that patient for 1 or more disease states. This follow-up care in an established patient would be considered incidental to the professional services of the physician, since the pharmacist is providing drug monitoring services that the physician would have personally performed. Delegation via a CPA meets the requirement of conforming to state laws, if the pharmacist is practicing in a state that has legislation expressly authorizing CPAs.

\section{Level of Service}

A misconception by many in the health care industry regards what level of service may be billed for the activities furnished by a pharmacist under a signed CPA. As previously mentioned, it is commonly thought that only a CPT 99211 code can be used to bill for pharmacist-provided incident-to services, but this is not true. If a pharmacist was personally submitting a bill, as the billing provider, that pharmacist would be required to only bill with 99211, since the pharmacist is not a recognized Medicare provider. However, since the pharmacist is not the billing provider when providing clinical services in an incident-to model, the level of service billed is directly related to the level of services rendered and the documentation of those services. This model is no different than what is currently used and accepted when nonphysician providers (NPPs), such as nurse practitioners or advanced registered nurse practitioners, work under an incident-to model. The level of the bill is commensurate with the level of service provided, as long as that service is consistent with what the NPP has been authorized to perform by the physician. While NPPs may also be considered billable providers in the sense that they may independently bill an insurance carrier on their own, when they are working within an incident-to mode, the supervising physician is the billing provider.

As previously mentioned, the definition of incident-to states that "services are billed as Part B services to your carrier as if you [the physician] personally provided them." Typically, a physician would not bill for complex medication management services using CPT 99211 (Level 1) nor would an NPP when providing the same level of service. If the services provided by the pharmacist are within the state scope of practice and align with the billing requirements for a given level of service, then they are eligible to be billed at a level higher than 99211. Review of the CPT E/M codes 99211-99215, as well as the requirements for documentation (history, exam, and medical decision making) show that the necessary elements of billing are well within the scope of practice for a pharmacist, especially when those services are outlined in a CPA. Therefore, pharmacists providing services in an incident-to model are eligible to capture higher levels of reimbursement when the supervising physician submits the bill for the professional services of the pharmacist. This eligibility is predicated upon the following: (a) the services provided by the pharmacist are within the scope and practice, (b) all other incident-to requirements are met, and (c) the service provided and accompanying documentation meet the billing requirements for the level of bill submitted.

\section{Drawbacks of Incident-to Billing}

There may be downsides of incident-to billing to consider. First, the supervising physician must provide an attestation to the pharmacist's documentation that the physician was present in the clinic at the time the services were rendered. However, this attestation is the same that is required when a physician supervises an NPP performing incident-to services and is often only 1 sentence in length. Therefore, the time required to document the attestation is minimal, and a new workflow is not required. Second, incident-to billing is likely not applicable to pharmacists working in a retail setting. Applicable services 
are likely best provided within a physician's office in order to provide collaborative care and ensure that the physician is maintaining ongoing involvement in the care of the patient. There may also be burdens associated with the development and maintenance of CPAs, but after the initial development process, the time required to review and update the CPA should be minimal.

\section{Conclusions}

Medicare publicly supports the performance of clinical services by pharmacists in an incident-to manner. Despite the lack of formal recognition of pharmacists as providers at the federal level, most states have language authorizing CPAs, which provide statutory authority at the state level for the provision of clinical services by pharmacists. When those services are provided under the direct supervision of a physician, then they are eligible to be billed as incident-to by the supervising physician. While national pharmacy organizations push towards formal recognition as providers, using incident-to billing in the interim allows the services performed by pharmacists to be billed at a higher level, vastly improving the financial sustainability of clinical pharmacy services and increasing the incorporation of pharmacists into care teams, which has been shown to improve clinical outcomes, increase patient access to care, and reduce health care spending.

\section{Authors}

ERIC DIETRICH, PharmD, BCPS, CPC-A, and JOHN G. GUMS, PharmD, FCCP, University of Florida College of Pharmacy, Gainesville.

AUTHOR CORRESPONDENCE: Eric Dietrich, PharmD, BCPS, CPC-A, Clinical Assistant Professor, University of Florida College of Pharmacy, Gainesville, FL 32610. Tel.: 352.294.5648;

E-mail:ead1129@ufl.edu.

\section{DISCLOSURES}

This work was not supported by any funding source. The authors have no relevant conflicts of interest to disclose.

\section{REFERENCES}

1. Hwang AY, Gums TH, Gums JG. The benefits of physician-pharmacist collaboration. J Fam Pract. 2017;66(12):El-E8

2. Cranor CW, Bunting BA, Christensen DB. The Asheville Project: longterm clinical and economic outcomes of community pharmacy diabetes care program. J Am Pharm Assoc (Wash). 2003;43(2):173-84.

3. Bunting BA, Smith BH, Sutherland SE. The Asheville Project: clinical and economic outcomes of a community-based long-term medication therapy management program for hypertension and dyslipidemia. J Am Pharm Assoc (2003). 2008;48(1):23-31

4. Garrett DG, Bluml BM. Patient self-management program for diabetes: first-year clinical, humanistic, and economic outcomes. J Am Pharm Assoc (2003). 2005;45(2):130-37.

5. Fera T, Bluml BM, Ellis WM, Schaller CW, Garrett DG. The Diabetes Ten City Challenge: interim clinical and humanistic outcomes of a multisite community pharmacy diabetes care program. J Am Pharm Assoc (2003). 2008;48:181-90

6. Fera T, Bluml BM, Ellis WM. The Diabetes Ten City Challenge: final economic and clinical results. J Am Pharm Assoc (2003). 2009;49(3):383-91.

7. Giberson S, Yoder S, Lee MP. Improving patient and health system outcomes through advanced pharmacy practice: a report to the U.S. Surgeon General. Office of the Chief Pharmacist. U.S. Public Health Service. December 2011. Available at: https://www.accp.com/docs/positions/misc/ improving_patient_and_health_system_outcomes.pdf. Accessed September 24, 2018.

8. Center for Medicaid \& CHIP Services. State flexibility to facilitate timely access to drug therapy by expanding the scope of pharmacy practice using collaborative practice agreements, standing orders or other predetermined protocols. CMCS informational Bulletin. January 17, 2017. Available at: https://www.medicaid.gov/federal-policy-guidance/downloads/cib011717. pdf. Accessed September 24, 2018.

9. Medicare Learning Network. "Incident-to" services. MLN Matters, number SE0441. Updated August 23, 2016. Available at: https://www. cms.gov/Outreach-and-Education/Medicare-Learning-Network-MLN/ MLNMattersArticles/downloads/se0441.pdf. Accessed September 24, 2018

10. Centers for Medicare \& Medicaid Services. Chapter 15: Covered medical and other health services. In: Medicare Benefit Policy Manual. Updated February 2, 2018. Available at: https://www.cms.gov/Regulations-andGuidance/Guidance/Manuals/downloads/bp102c15.pdf. Accessed September 24, 2018.

11. American Academy of Family Physicians. AAFP, CMS clarify "incident to" rules relating to pharmacists' services. News release. April 16, 2014. Available at: https://www.aafp.org/news/practice-professionalissues/20140416incidenttoltr.html. Accessed September 24, 2018.

12. Centers for Disease Control and Prevention. Advancing team-based care through collaborative practice agreements: a resource and implementation guide for adding pharmacists to the care team. 2017. Available at: https://www.cdc.gov/dhdsp/pubs/docs/CPA-Team-Based-Care.pdf. Accessed September 24, 2018. 